\title{
CONNECTIONS ON SEMISIMPLE LIE GROUPS
}

\author{
BY
}

\author{
ROBERT E. BECK
}

\begin{abstract}
The plus and minus connections of Cartan and Schouten, which exist on any Lie group, have the following three properties: (1) the connection is left invariant, (2) the curvature of the connection is zero, (3) the set of maximal geodesics through the identity of the Lie group is equal to the set of one-parameter subgroups of the Lie group. It is shown that the plus and minus connections are the only ones with these properties on a real simple Lie group. On a real semisimple Lie group the connections with these properties are in one-to-one correspondence with the ways of choosing an ideal of the Lie algebra and then choosing a complementary subspace to it.
\end{abstract}

1. Introduction. To find all the connections on a real semisimple Lie group with the properties listed in the abstract, we restate the properties in terms of representations of Lie algebras and classify the representations which correspond to connections. Specifically, these properties induce skew-symmetric representations of the corresponding Lie algebra. By using computational methods of representation theory, the skew-symmetric representations of a complex simple Lie algebra can be determined. With standard techniques of representation theory these results are extended to complex semisimple Lie algebras in $\S 3$ and then to real semisimple Lie algebras in $\$ 4$.

Let $G$ be a real Lie group with $T_{e}(G)$, the tangent space at the identity, considered as its Lie algebra $g$. Let $\nabla$ be a left invariant affine connection on $G$. Nomizu [4] has shown that $\nabla$ arises from a bilinear map $\alpha: g \times g \rightarrow g$. When the curvature of $\nabla$ is zero, it is easily shown that $\alpha$ is a Lie algebra representation of $g$ on itself, considering $\alpha: g \rightarrow \mathrm{gl}(g)$. Helgason [2] shows that the geodesics of $\nabla$ have property (3) if $\alpha$ is skew-symmetric. Consequently, the desired connections arise from representations $(\rho, g)$ of $g$ on itself which are skew-symmetric $(\rho(x) x=0$ for all $x \in g$ ).

Let $g$ be a complex semisimple Lie algebra and let $h$ be a Cartan subalgebra of $g$. Let $\Delta$ be the set of roots of $g$ with respect to $h$ and let $\pi=\left\{\alpha_{1}, \ldots, \alpha_{n}\right\}$ be a set of simple roots of $\Delta$. Let $\left\{H_{1}, \ldots, H_{n}\right\}$ be a basis of $h$ chosen so that $\alpha_{i}\left(H_{j}\right) \in \boldsymbol{Q}$, the field of rational numbers, for $i, j=1, \ldots, n$. In this paper, we will call an element $H \in h$ generic if $H=\sum_{i=1}^{n} a_{i} H_{i}$ where the complex coefficients $a_{1}, \ldots, a_{n}$ are linearly independent over $\boldsymbol{Q}$. It can be shown that generic elements have the properties listed in the following lemma.

Presented to the Society, January 29, 1969 under the title On counting connections on Lie groups and May 12, 1969; received by the editors June 24, 1970.

AMS 1970 subject classifications. Primary 22E15, $17 \mathrm{~B} 10$.

Key words and phrases. Real semisimple Lie group, affine connection, real semisimple Lie algebra, skew-symmetric representation. 
LEMMA 2. (a) $\alpha(H) \neq 0$ for every root $\alpha$ of $g$ and every generic element of $h$.

(b) If $\omega, \omega^{\prime}$ are weights of some arbitrary representation $(\sigma, V)$ of $g$, and if $\omega(H)=\omega^{\prime}(H)$ for some generic element of $h$, then $\omega=\omega^{\prime}$.

(c) Let $(\sigma, V)$ be a representation of $g$. Assume that for a generic element, $H$, of $h$, there is a nonzero $v \in V$ and $a \omega \in h^{*}$ such that $\sigma(H) v=\omega(H) v$. Then $\omega$ is a weight of $(\sigma, V)$.

2. Skew-symmetric representations. Throughout this section let $(\rho, g)$ be a skew-symmetric representation of $g$. Let $V_{0}=\{x \in g \mid \rho(g) x=0\}$. Using the definition of skew-symmetry it is easy to show that

$$
(\rho(x))^{2}=\rho(x) \text { ad } x \text { for all } x \in g
$$

and that $\operatorname{ker} \rho=V_{0}$. Hence, $V_{0}$ is an ideal in $g$.

LEMMA 1. If $\rho$ is a faithful skew-symmetric representation of a semisimple Lie algebra, then every invariant subspace is an ideal.

Proof. Let $V_{1}$ be an invariant subspace of $g$ under $\rho$. Since any representation of a semisimple Lie algebra is completely reducible [3, p. 79], we may write $g=V_{1} \oplus V_{2}$ where $V_{2}$ is an invariant subspace of $g$ under $\rho$. Now $\rho(g)[x, V] \subseteq V_{1}$ where $x \in g$ and $v \in V_{1}$. Writing $[x, v]=[x, v]_{1}+[x, v]_{2}$ where $[x, v]_{i} \in V_{i}, i=1,2$, we have that

$$
V_{1} \supseteq \rho(g)[x, v]=\rho(g)\left([x, v]_{1}\right)+\rho(g)\left([x, v]_{2}\right) .
$$

From the invariance of $V_{i}, i=1,2$, we conclude that $[x, v]_{2}=0$ and that $V_{1}$ is an ideal.

We are now able to characterize the weights of a skew-symmetric representation of a complex semisimple Lie algebra.

THEOREM 3. Let $g$ be a complex semisimple Lie algebra. Every nonzero weight of $\rho$ is a root of $g$. Furthermore, 0 is a weight of $\rho$.

Proof. With respect to ad we may write

$$
g=g(0)+\sum_{\alpha} g(\alpha), \text { where } \alpha \text { is a root of } g .
$$

With respect to $(\rho, g)$ we may write

$$
g=V(0)+\sum_{\omega} V(\omega), \quad \text { where } \omega \text { is a weight of }(\rho, g) \text {. }
$$

We first show that $g(0)$, which is a Cartan subalgebra, $h$, of $g$ is a subspace of $V(0)$. Let $Y \in h$. By equation (1) for all $H \in h$ we have $(\rho(H))^{2} Y=\rho(H)[H, Y]=0$. It can be shown that for all $H \in h, \rho(H)$ is semisimple. Thus, $(\rho(H))^{2} Y=0$ implies that $\rho(H) Y=0$ and $Y \in V(0)$. Hence, 0 is a weight of $\rho$.

Now let $H \in h$ be generic. Then $\alpha(H) \neq 0$ for every root $\alpha$ of $g$. Let $g(\alpha)=C E_{\alpha}$ and consider $\rho(H) E_{\alpha}$.

$$
\rho(H)\left(\rho(H) E_{\alpha}\right)=\rho(H)\left[H, E_{\alpha}\right]=\alpha(H) \rho(H) E_{\alpha} .
$$


There are two cases:

(a) $\rho(H) E_{\alpha}=0$ which, by Lemma 2, choosing $\omega=0$, implies that $E_{\alpha} \in V(0)$.

(b) $\rho(H) E_{\alpha} \neq 0$ which, by Lemma 2 and equation (2), implies that $\alpha$ is a weight and $\rho(H) E_{\alpha} \in V(\alpha)$.

Label the roots so that (a) is true for $\alpha^{(1)}, \ldots, \alpha^{(k)}$ and (b) is true for $\alpha^{(k+1)}, \ldots$, $\alpha^{(r)}$. Then

$$
\begin{gathered}
V(0) \supseteq h+g\left(\alpha^{(1)}\right)+\cdots+g\left(\alpha^{(k)}\right), \\
V\left(\alpha^{(j)}\right) \supseteq \rho(H) g\left(\alpha^{(j)}\right), \quad j=k+1, \ldots, r .
\end{gathered}
$$

By a dimension argument, we have equality in (3) and (4). Therefore,

$$
g=V(0)+V\left(\alpha^{(k+1)}\right)+\cdots+V\left(\alpha^{(r)}\right)
$$

is a weight space decomposition of $g$ and $\alpha^{(k+1)}, \ldots, \alpha^{(r)}$ are the only nonzero weights of $(\rho, V)$.

Knowing about the weights of $(\rho, g)$ where $g$ is complex semisimple, we can prove this theorem about equivalent representations.

THEOREM 4. Let $g$ be complex semisimple. If $\rho$ is equivalent to ad, then $\rho=\mathrm{ad}$.

Proof. There is an element $B \in \mathrm{GL}(g)$ such that, for all $X \in g$, ad $X=B^{-1} \rho(X) B$. Since $\rho$ skew-symmetric, we have $B$ ad $X B^{-1}=0$ for all $X \in g$, and using the nonsingularity of $B,\left[X, B^{-1} X\right]=0$. By polarization, $\left[X, B^{-1} Y\right]=\left[B^{-1} X, Y\right]$, or $[B X, Y]=[X, B Y]$ for all $X, Y \in g$. Since $\rho$ is equivalent to ad, $h \subseteq V(0)$ implies that $h=V(0)$.

Let $H$ be a generic element of $h$. We have $\rho(H) B E_{\alpha}=B$ ad $H E_{\alpha}=\alpha(H) B E_{\alpha}$ for every root, $\alpha$, of $g$. Since $H$ is generic, $B E_{\alpha} \in V(\alpha)$ and, from the proof of Theorem 3, $\rho(H) E_{\alpha} \in V(\alpha)$. Therefore, there is a nonzero constant depending on $\alpha$ and $H$ which will be denoted by $C(\alpha, H)$ such that $B E_{\alpha}=C(\alpha, H) \rho(H) E_{\alpha}$.

For any root $\alpha$ and any $H \in h$, equation (1) implies that

$$
(\rho(H))^{2} E_{\alpha}-\rho(H)\left[H, E_{\alpha}\right]=\rho(H)\left(\rho(H) E_{\alpha}-\alpha(H) E_{\alpha}\right)=0 .
$$

Therefore, if $H$ is a generic, $\rho(H) E_{\alpha}-\alpha(H) E_{\alpha}=H^{(\alpha)} \in h$. Let $\beta$ be any root of $g$. Then for generic $H$,

$$
\left[B E_{\alpha}, E_{\beta}\right]=\left[C(\alpha, H) \rho(H) E_{\alpha}, E_{\beta}\right]=C(\alpha, H) \alpha(H)\left[E_{\alpha}, E_{\beta}\right]+C(\alpha, H) \beta\left(H^{(\alpha)}\right) E_{\beta}
$$
and

$$
\left[E_{\alpha}, B E_{\beta}\right]=\left[E_{\alpha}, C(\beta, H) \rho(H) E_{\beta}\right]=C(\beta, H) \beta(H)\left[E_{\alpha}, E_{\beta}\right]-C(\beta, H) \alpha\left(H^{(\beta)}\right) E_{\alpha} .
$$

But $\left[B E_{\alpha}, E_{\beta}\right]=\left[E_{\alpha}, B E_{\beta}\right]$ which gives us on equating terms

$$
(C(\alpha, H) \alpha(H)-C(\beta, H) \beta(H))\left[E_{\alpha}, E_{\beta}\right]=0
$$

and

$$
C(\alpha, H) \beta\left(H^{(\alpha)}\right) E_{\beta}+C(\beta, H) \alpha\left(H^{(\beta)}\right) E_{\alpha}=0 .
$$

If $\alpha=\beta$, we have $\alpha\left(H^{(\alpha)}\right)=0$. If $\alpha \neq \beta$, we have $\beta\left(H^{(\alpha)}\right)=0$ from (6). 
Therefore, for any root $\alpha, H^{(\alpha)}=0$, and $\rho(H) E_{\alpha}=$ ad $H E_{\alpha}$ for all generic $H \in h$. Consequently $B E_{\alpha}=C(\alpha, H) \alpha(H) E_{\alpha}$ for all roots $\alpha$. Since $\rho(H) H^{\prime}=0=\operatorname{ad} H H^{\prime}$ for all $H, H^{\prime} \in h$ and by skew-symmetry $\rho\left(E_{\alpha}\right) H=\operatorname{ad} E_{\alpha} H$ for all roots $\alpha$ and all generic elements of $h$, to show $\rho(X)=\operatorname{ad} X$ for all $X$ we must only show $\rho\left(E_{\alpha}\right) E_{\beta}$ $=\operatorname{ad} E_{\alpha} E_{\beta}$ for $\alpha \neq \beta$. Now

$$
\rho\left(E_{\alpha}\right) E_{\beta}=B \operatorname{ad} E_{\alpha} B^{-1} E_{\beta}=B\left[E_{\alpha}, E_{\beta}\right] / C(\beta, H) \beta(H) .
$$

If $\alpha+\beta$ is not a root, then $\rho\left(E_{\alpha}\right) E_{\beta}=0=\operatorname{ad} E_{\alpha} E_{\beta}$. Otherwise $\left[E_{\alpha}, E_{\beta}\right]=k E_{\alpha+\beta}$ where $k$ is a constant and

$$
\rho\left(E_{\alpha}\right) E_{\beta}=(C(\alpha+\beta, H)(\alpha+\beta)(H) / C(\beta, H) \beta(H)) \text { ad } E_{\alpha} E_{\beta} .
$$

But from (5), $C(\beta, H) \beta(H)=C(-\beta, H)(-\beta(H))$ since $\left[E_{\alpha}, E_{\beta}\right] \neq 0$ and $C(-\beta, H)$ $\times(-\beta(H))=C(\alpha+\beta, H)(\alpha+\beta)(H)$ since $\left[E_{\alpha+\beta}, E_{-\beta}\right] \neq 0$. Therefore, $\rho\left(E_{\alpha}\right) E_{\beta}=$ ad $E_{\alpha} E_{\beta}$ and, by linearity, $\rho(X)=\operatorname{ad} X$ for all $X \in g$.

3. Skew-symmetric representations of complex semisimple Lie algebras. In this section we find all the skew-symmetric representations of a complex semisimple Lie algebra. The technique used is to first study skew-symmetric representations of complex simple Lie algebras and then extend these results to the semisimple case. Throughout this section $g$ will be complex semisimple and $(\rho, g)$ will be skewsymmetric.

Initially, our goal is to show that the only nonzero skew-symmetric representation of a complex simple Lie algebra is the adjoint representation. Since $V_{0}$ is an ideal, when $g$ is simple a skew-symmetric representation of $g$ will be either 0 or irreducible and faithful. If $\rho \neq 0$, in light of Theorem 4 , we need only prove that $\rho$ is equivalent to ad, or equivalently the highest weight of $\rho$ is equal to highest root of $\mathrm{g}$. We then will be able to conclude

THEOREM 5. If $g$ is simple, $\rho=0$ or $\rho=\mathrm{ad}$.

Lemma 6. If $g$ is simple and $\rho \neq 0$, then the highest weight of $\rho$ is equal to the highest root of $g$.

Proof. We first consider the case that all the roots of $g$ have the same length, i.e. $\Delta$ is simply laced $\left(g=A_{n}, D_{n}, E_{n}\right)$. Let $\Lambda=$ set of weights of $\rho$. Let $\omega \in \Lambda$ and $\alpha \in \Delta$. By Theorem 3, $\omega \in \Delta$. Hence, there is an element $s$ of the Weyl group of $g$ such that $s(\omega)=\alpha$. But $s(\omega) \in \Lambda$ since the Weyl group preserves weights. Therefore $\Lambda=\Delta$, and the highest weight equals the highest root.

Now if the roots of $g$ have two different lengths, i.e. $\Delta$ is doubly laced, consider the case where the length of the highest weight $=$ the length of the highest root. By the same argument as above, one can show that the highest root is a weight, and, therefore, must be the highest weight.

Finally, if the length of the highest weight $\neq$ the length of the highest root, the same argument shows that the highest weight $=$ the highest root with length not 
equal to the length of the highest root of $g$. In fact, the highest root of a doubly laced root system always has the longer length. The highest root of short length is the highest weight of an irreducible representation of $g$, but in each case $\operatorname{dim}(\rho)$ $<\operatorname{dim}(g)$ which is contrary to hypothesis. Consequently, this situation is not possible, and the other two cases give the desired result.

By examining the possible dimensions for irreducible representations, the author has also proved Lemma 6 in the following form.

Lemma $6^{\prime}$. Let $(\sigma, V)$ be an irreducible representation of a complex simple Lie algebra $g$ such that $\operatorname{dim}(\sigma)=\operatorname{dim}(g)$. Then $\sigma \sim \operatorname{ad}$.

Turning to the semisimple case we first consider representations for which

$$
V_{0}=\{x \mid \rho(g) X=0\}
$$

is zero. It has been shown that with this hypothesis $\rho$ is faithful and every invariant subspace is an ideal. The converse is also true.

Lemma 7. Let $g$ be semisimple and $(\rho, g)$ be skew-symmetric. If $V_{0}=0$, every ideal of $g$ is an invariant subspace of $\rho$.

Proof. Let $g=V_{1} \oplus \cdots \oplus V_{s}$ where $V_{j}, j=1, \ldots, s$, are irreducible invariant subspaces of $g$. Then $V_{j}$ is an ideal for each $j$. If each $V_{j}$ is a simple ideal, we are finished, so suppose that $V_{k}$ is not simple. Then $\rho$ restricted to $V_{k}$ is irreducible, faithful, and skew-symmetric. Let $V_{k}=g_{1} \oplus \cdots \oplus g_{r}$ where the $g_{i}$ are simple ideals of $g$. Let $\rho_{i}=\rho \mid g_{i}$. Using the same method as in the proof of Theorem 3, one may show that every nonzero weight of $\rho_{i}$ is a root of $g_{i}$. Since $\rho$ is irreducible it may be written as the tensor sum of the representations $\sigma_{i}$ where $\sigma_{i}: g_{i} \rightarrow \mathrm{gl}\left(W_{i}\right)$ and $W_{i}$ is a minimal $\rho_{i}$ invariant subspace of $g$. That is, for $\rho$ restricted to $V_{k}$

$$
\rho\left(X_{1}+\cdots+X_{r}\right)\left(w_{1} \otimes \cdots \otimes w_{r}\right)=\sum_{i=1}^{r} w_{1} \otimes \cdots \otimes \rho\left(X_{i}\right) w_{i} \otimes \cdots \otimes w_{r}
$$

for $X_{i} \in g_{i}$, and $w_{i} \in W_{i}$. Every weight of $\rho$ is the sum of weights of $\sigma_{i}$. But weights of $\sigma_{i}$ are also weights of $\rho_{i}$ and hence roots of $g_{i}$. Therefore, we have on one hand every weight of $\rho$ is a root of $g$, and on the other hand every weight of $\rho$ is the sum of roots $g_{i}$ and hence not a root of $g$. This contradicts the assumption that $V_{k}$ was not simple.

THEOREM 8. If $(\rho, g)$ is a skew-symmetric faithful representation of a complex semisimple Lie algebra $g$, then $\rho=$ ad.

Proof. Let $g=g_{1} \oplus \cdots \oplus g_{k}$ where $g_{j}$ is a simple ideal. Write $\rho=\rho_{1}+\cdots+\rho_{k}$ where $\rho_{j}$ is an irreducible representation of $g$ on $g_{j}$. Let $\sigma_{j}$ be equal to $\rho_{j}$ restricted to $g_{j}$. Then $\sigma_{j}$ is skew-symmetric and faithful. From Theorem $5, \sigma_{j}=\mathrm{ad}_{g_{j}}$ and hence $\rho=\operatorname{ad}_{g}$. 
We now consider the case where $V_{0} \neq 0$. We have seen that $V_{0}$ is an invariant subspace under $\rho$. Since $g$ is semisimple we may write $g=V_{0} \oplus V_{1}$ as vector spaces where $V_{1}$ is a complementary invariant subspace to $V_{0}$ and also $g=g_{1} \oplus g_{2}$ where $g_{1}=V_{0}$ and $g_{2}$ is an ideal. Then as vector spaces $V_{1} \cong g_{2}$. Let $\pi_{i}: g \rightarrow g_{i}, i=1,2$, be projection maps. Then $\pi_{2}$ is the isomorphism between $V_{1}$ and $g_{2}$. Also if we let $\phi=\pi_{1} \circ \pi_{2}^{-1}$, then $\phi: g_{2} \rightarrow g_{1}$ and

$$
V_{1}=\left\{x+\phi(x) \mid x \in g_{2}\right\} .
$$

We see that $\phi$ is a vector space homomorphism, and it is a Lie algebra homomorphism if and only if $V_{1}$ is a subalgebra of $g$.

Now let $\rho_{1}$ be the representation of $g_{2}$ on $V_{1}$ induced by restricting $\rho$ to $g_{2}$. Then $\rho_{1}$ is faithful. For if $\rho_{1}(x)=0$ for some $x \in g_{2}$, we have $\rho_{1}(x) v_{1}=\rho(x) v_{1}=\rho\left(v_{1}\right) x=0$ for all $v_{1} \in V_{1}$. Furthermore, $\rho(x) v_{0}=\rho\left(v_{0}\right) x=0$ for all $v_{0} \in V_{0}$. Therefore, $\rho(v) x=0$ for all $v=v_{0}+v_{1} \in g$ which implies that $x \in g_{1}$ and consequently that $x=0$.

Let $\rho_{2}(x)=\pi_{2} \circ \rho_{1}(x) \circ \pi_{2}^{-1}$ where $x \in g_{2}$. Then $\rho_{2}$ is a faithful representation of $g_{2}$ on itself. Also we have

$$
\rho_{2}(x) x=\pi_{2}\left(\rho_{1}(x)(x+\phi(x))\right)=\pi_{2}(\rho(x)(x)+\rho(x) \phi(x))=0 .
$$

From Theorem 5 we conclude that $\rho_{2}(x) y=[x, y]$ and that

$$
\rho(x+\phi(x))(y+\phi(y))=[x, y]+\phi([x, y])
$$

where $x, y \in g_{2}$. We have shown

THEOREM 9. Let $(\rho, g)$ be a skew-symmetric representation of a complex semisimple Lie algebra of whose zero space is $g_{1}$. Let $V_{1}$ be a complementary subspace to $g_{1}$ defined by $\phi: g_{2} \rightarrow g_{1}$ where $g_{2}$ is a complementary ideal to $g_{1}$. Then for all $x, y \in g_{2}$, we have $\rho(x+\phi(x))(y+\phi(y))=[x, y]+\phi([x, y])$ and $\left.\rho\right|_{V_{1}}=\left.\operatorname{ad}\right|_{V_{1}}$ if $V_{1}$ is a subalgebra of $g$.

4. Skew-symmetric representations of real semisimple Lie algebras. In this section we extend the results of the preceding section to real semisimple Lie groups. We use the standard techniques of complexifying real Lie algebras and their representations which are described below.

If $V$ is a vector space over $R$, the field of real numbers, denote by $V^{c}$ the complex vector space obtained from $V$ by extending the ground field. That is,

$$
V^{c}=V \otimes_{R} C \cong V \oplus i V \text { and } \operatorname{dim}_{R} V=\operatorname{dim}_{C} V^{c} .
$$

If $g$ is a real Lie algebra, then $g^{c}$ is a complex Lie algebra, called the complexification of $g$, and the bracket product in $g^{c}$ is defined by

$$
[X+i Y, Z+i W]=([X, Z]-[Y, W])+i([Y, Z]+[X, W]) .
$$

It can be shown that $g$ is semisimple if and only if $g^{c}$ is semisimple. 
If $(\rho, V)$ is a real representation of a real Lie algebra $g$, denote by $\left(\rho^{c}, V^{c}\right)$ the complex representation of $g$ induced by $\rho$ where

$$
\rho^{c}(X)(v+i w)=\rho(X) v+i \rho(X) w
$$

for any $X \in g$ and $v+i w \in V^{c}$. $\left(\rho^{c}, V^{c}\right)$ can be extended to a representation of $g^{c}$, denoted by $\left(P, V^{c}\right)$ and defined by

$$
P(X+i Y)=\rho^{c}(X)+i \rho^{c}(Y)
$$

for all $X+i Y \in g^{c}$.

The properties of faithfulness, equivalence, skew-symmetry, and adjoint all lift from a real representation of a real Lie algebra to the induced representation of the complexification of the algebra, and conversely they are induced on a real representation of a real Lie algebra by its lift to the complexification of the algebra. Consequently, we may extend Theorem 8 to read

THEOREM 10. If $(\rho, g)$ is a skew-symmetric faithful representation of a real semisimple Lie algebra, $g$, then $\rho=\mathrm{ad}$.

Using Theorem 10 in place of Theorem 8 we may extend Theorem 9 to read

THEOREM 11. Let $(\rho, g)$ be a skew-symmetric representation of a real semisimple Lie algebra $g$ whose zero-space is $g_{1}$. Let $V_{1}$ be a complementary subspace to $g_{1}$ defined by $\phi: g_{2} \rightarrow g_{1}$ where $g_{2}$ is a complementary ideal to $g_{1}$. Then for all $x, y \in g_{2}$, we have

$$
\rho(x+\phi(x))(y+\phi(y))=[x, y]+\phi([x, y]) .
$$

Converting the Lie algebra results to Lie group ones as described in $\$ 1$, we have shown

THEOREM 12. For a real semisimple Lie group $G$, the left invariant linear connections on $G$ which have

(a) zero curvature, and

(b) set of maximal geodesics through the identity = set of one-parameter subgroups, are in one-to-one correspondence with the decompositions of the Lie algebra $g$ of $G$ into the vector space sum of an ideal $g_{1}$ and a subspace $V_{1}$ where the representation defining the connection is zero on $g_{1}$ and as in Theorem 11 on $V_{1}$.

However, if we assume that the connection is also right invariant, we can show

THEOREM 13. The bi-invariant connections on a real semisimple Lie group $G$ which have properties (a) and (b) of Theorem 12 are in one-to-one correspondence with the decompositions of the Lie algebra $g$ of $G$ into the direct sum of two ideals, $g=g_{1} \oplus g_{2}$, where the representation defining the connection is zero on $g_{1}$ and equal to the adjoint representation on $g_{2}$. 
Proof. Using Nomizu's results [4], we have that a bi-invariant connection satisfying (a), and (b) is defined by a skew-symmetric representation $(\rho, g)$ of $g$ such that

$$
\text { ad } X \rho(Y) Z=\rho(\operatorname{ad} X \cdot Y) Z+\rho(Y)(\operatorname{ad} X) Z .
$$

Applying this identity to the result of Theorem 11 yields $[X, \phi([Y, Z])]=0$ for all $X \in g_{1}$ and $Y, Z \in g_{2}$. Therefore, $\phi([Y, Z])=0$. But since $g_{2}$ is semisimple $\left[g_{2}, g_{2}\right]$ $=g_{2}$ and we have $\phi=0$. Therefore, given $g_{1}$, the only choice for $V_{1}$ is $g_{2}$.

The author would like to thank Professor M. Goto, his dissertation advisor, for his helpful suggestions on the material of this paper which is part of the author's Ph.D. dissertation written at the University of Pennsylvania, Spring 1969.

\section{BIBLIOGRAPHY}

1. E. Cartan and J. Schouten, On the geometry of the group-manifold of simple and semisimple groups, Nederl. Akad. Wetensch. Proc. Ser. A 29 (1926), 803-815.

2. S. Helgason, Differential geometry and symmetric spaces, Pure and Appl. Math., vol. 12, Academic Press, New York, 1962. MR 26 \#2986.

3. N. Jacobson, Lie algebras, Interscience Tracts in Pure and Appl. Math., no. 10, Interscience, New York, 1962. MR 26 \#1345.

4. K. Nomizu, Invariant affine connections on homogeneous spaces, Amer. J. Math. 76 (1954), 33-65. MR 15, 468.

Department of Mathematics, Villanova University, Villanova, Pennsylvania 19085 